\title{
Reciprocity Between Robustness of Period and Plasticity of Phase in Biological Clocks
}

\author{
Tetsuhiro S. Hatakeyama* and Kunihiko Kaneko \\ Department of Basic Science, The University of Tokyo, 3-8-1 Komaba, Meguro-ku, Tokyo 153-8902, Japan \\ (Received 26 May 2015; revised manuscript received 7 October 2015; published 19 November 2015) \\ Circadian clocks exhibit the robustness of period and plasticity of phase against environmental changes \\ such as temperature and nutrient conditions. Thus far, however, it is unclear how both are simultaneously \\ achieved. By investigating distinct models of circadian clocks, we demonstrate reciprocity between \\ robustness and plasticity: higher robustness in the period implies higher plasticity in the phase, where \\ changes in period and in phase follow a linear relationship with a negative coefficient. The robustness of \\ period is achieved by the adaptation on the limit cycle via a concentration change of a buffer molecule, \\ whose temporal change leads to a phase shift following a shift of the limit-cycle orbit in phase space. \\ Generality of reciprocity in clocks with the adaptation mechanism is confirmed with theoretical analysis of \\ simple models, while biological significance is discussed.
}

DOI: 10.1103/PhysRevLett.115.218101

PACS numbers: 87.18.Yt, 05.45.Xt, 87.18.Vf

Biological systems are both robust to external changes in the environment, and plastic to adapt to environmental conditions. How are the robustness and plasticity, which seem to be opposing properties at a first glance, compatible with each other? In the present Letter, we address this question, by focusing on biological clocks, which are ubiquitous in organisms.

Such biological clocks often work as pacemakers, to adapt to periodic events. One of the most prominent examples of such oscillators is a circadian clock $[1,2]$. To respond to periodic events, the following two criteria are generally imposed on a biochemical oscillator.

(1) Robustness of period: If the period of an oscillator strongly depends on external conditions, the oscillator would not accurately predict time. For example, if the period of a circadian clock is sensitive to temperature, the clock malfunctions depending on the temperature. To avoid such an error, the period of pacemakers should not be affected by external conditions such as temperature and nutrient compensation $[3,4]$.

(2) Plasticity of phase: The period of the circadian clock of most organisms is known not to correspond precisely with 24 hours [5], and biological clocks are entrained with the external 24-hr cycle [6], so that the phase difference between the two does not increase with time. This entrainment is also necessary to adapt an abrupt change in the environment that may cause temporal misalignment between the internal and external cycles. For such entrainment, plasticity of the phase of the internal clock against external stimuli, e.g., changes in temperature and/or brightness, is needed.

Published by the American Physical Society under the terms of the Creative Commons Attribution 3.0 License. Further distribution of this work must maintain attribution to the author(s) and the published article's title, journal citation, and DOI.
Indeed, biological clocks satisfy both robustness and plasticity to changes in factors such as temperature and nutrient conditions, which change in the daily cycle. For example, circadian clocks of in vivo Drosophila [7], Neurospora [8], and in vitro cyanobacteria $[9,10]$ show temperature compensation of a period and are entrained by cyclic temperature changes. Robustness of period is also important to stable entrainment since it can reduce the difference between the period of inner clock and external cycle. In spite of some studies discussing the compatibility between the two properties [7,11-13], however, little is known about the quantitative relationship between the two properties.

To answer how the robustness of the period and plasticity of phase are compatible with each other, we first analyze two major models of a circadian clock, i.e., the posttranslational oscillator (PTO) $[9,14,15]$ and transcriptiontranslation-based oscillator (TTO) [12,13,15], which consists only of protein-protein interactions and both transcription and translation processes, respectively. Without imposing any special mechanism, we demonstrate that biological clocks with robustness of period against changes in an environmental factor generally exhibit phase entrainment against the cyclic change of that factorreciprocity between the robustness of period and plasticity of phase: the plasticity increases with robustness.

For the PTO model, we adopt the KaiC allosteric model [16], for an in vitro cyanobacterial circadian clock system [9]. Here, the KaiC protein consists of six monomers, each of which has a phosphorylation site. The protein has active and inactive forms. Active (inactive) KaiC are phosphorylated (dephosphorylated) step by step, respectively. Phosphorylation reactions are facilitated with KaiA as an enzyme and dephosphorylation reactions spontaneously progress without an enzyme. $k_{p}$ and $k_{d p}$ denote the rate of phosphorylation and dephosphorylation of KaiC, 


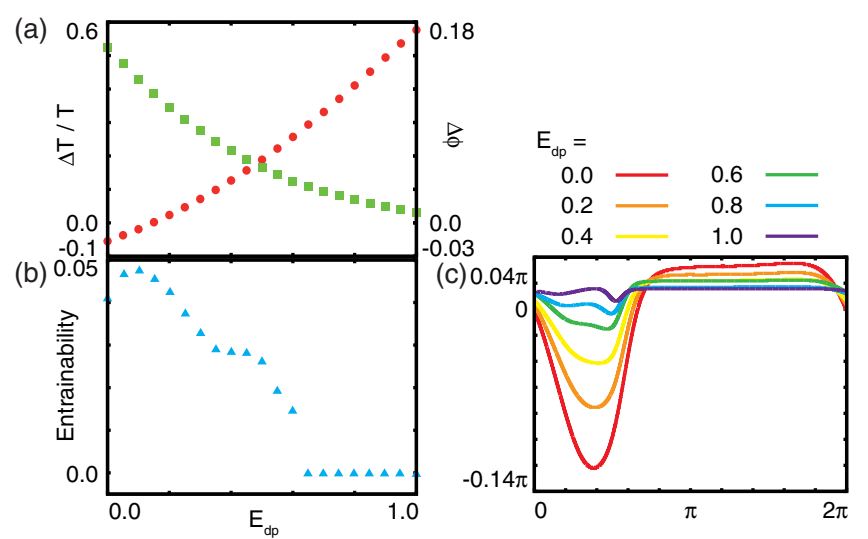

FIG. 1 (color online). Reciprocity between the robustness of period and plasticity of phase in the PTO model. (a) Difference between periods at two temperatures $\left(\beta_{1}=1.0\right.$ and $\left.\beta_{2}=1.5\right)$ $(\Delta T / T$, red circle) and the amplitude of the phase response curve against a transient jump of temperature from $\beta_{1}$ to $\beta_{2}$ ( $\Delta \phi$, green square) plotted against different values of $E_{d p}$ while $E_{p}$ is fixed at 1.0. $\Delta T$ is normalized by the period at $\beta_{1}$, and $\Delta \phi$ is normalized by the duration of stimulus and difference between $\beta_{1}$ and $\beta_{2}$. $\Delta T / T$ and $\Delta \phi$ are negatively correlated across the entire range of $E_{d p}$. (b) Entrainability is plotted against various $E_{d p}$ with fixed $E_{p}=1.0$. (c) Phase response curve against transient increase in $\beta$. As a stimulus, the inverse temperature $\beta$ is increased from $\beta_{1}$ to $\beta_{2}$ for the duration of one unit of time. Lines of different colors represent the PRCs for different values of activation energy for the dephosphorylation reaction $E_{d p}$.

respectively, which depend on temperature as $k_{p} \propto$ $\exp \left(-\beta E_{p}\right)$ and $k_{d p} \propto \exp \left(-\beta E_{d p}\right)$, where $E_{p}\left(E_{d p}\right)$ is the activation energy for phosphorylation (dephosphorylatiion), respectively, and $\beta$ is the inverse temperature by taking the Boltzmann constant as unity. The temporal evolution of the concentration of each phosphorylated active (inactive) $\mathrm{KaiC}$ is given by rate equations (see model equations and Fig. 1(a) of Ref. [17]).

This model shows a limit-cycle attractor in which the total phosphorylation level, i.e., the ratio of phosphorylated monomers, oscillates in time. We demonstrated that the robustness of the period against various environmental changes is achieved by enzyme-limited competition $[18,19]$ : With the increase in temperature, the abundance of the active form of the KaiC molecule increases, which in turn decreases the abundance of the free KaiA molecule, and thus the increase in the rate of phosphorylation $k_{p}$ is canceled out, when the total KaiA amount, $A_{\text {total }}$, is sufficiently small. This robustness in the period is achieved when $E_{p}$ is sufficiently larger than $E_{d p}$. We use the difference in periods between two different temperature conditions $(\Delta T / T)$ as an indicator of the robustness of period. Its dependence upon $E_{d p}-E_{p}$ is given in Fig. 1(a).

This clock, on the other hand, entrains against external periodic change, so that the phase of the phosphorylation oscillator coincides with that of the external cycle. By imposing an external periodic change in temperature, we computed how many cycles are needed for the clock to entrain with this external cycle, and defined entrainability as the inverse of the number (see Ref. [17]). Dependence of the entrainability and $\Delta T / T$ upon $E_{d p}$ with fixed $E_{p}$ is plotted in Figs. 1(a) (red circle) and 1(b). As $E_{d p}-E_{p}$ is smaller, $\Delta T / T$ becomes smaller and the entrainability is higher. In other words, if the period of the clock is more robust against temperature change, it is entrained faster with the external temperature cycle; i.e., the phase has higher plasticity.

Although this demonstrated the correlation between period robustness and phase plasticity, the entrainability here is a complicated indicator for the latter, as it can depend on the form of external cycle. Hence, we introduce a more tractable indicator for the plasticity of phase, by using a phase response curve (PRC) [20]. A PRC is a function of phase and represents a phase shift introduced by a transient stimulus. When a transient stimulus is added to an oscillatory system, the period of oscillation is temporally altered depending on the phase when the stimulus was added. The period finally returns to its original value. In this time, the phase of the oscillator progresses (or is delayed) from the original phase because of the temporal shortening (or lengthening) of the period. The PRC represents such a phase shift $\Delta \phi$ as a function of the phase $\phi$ when the stimulus is applied. We computed the PRC by transiently changing the inverse temperature from $\beta_{1}$ to $\beta_{2}$ for one time unit [see Fig. 1(c)], by defining the phase of oscillation by the time when the total phosphorylation level takes the maximum value at $\phi=0,2 \pi, \ldots$ As an indicator of the plasticity of phase, we measured the difference between maximum and minimum values of the phase change $\Delta \phi$ in PRC [21] normalized by the magnitude of a stimulus by fixing its duration as one time unit. The dependence of $\Delta \phi$ and $\Delta T / T$ on $E_{d p}$ with fixed $E_{p}$ is plotted in Fig. 1(a). When $E_{d p}$ is low, i.e., when the temperature dependence of dephosphorylation reaction is weak, $\Delta T / T$ is small and $\Delta \phi$ is large. This reciprocity was also obtained against changes in other parameters, $\beta_{1}$ and $A_{\text {total }}$ (see Fig. 3 of Ref. [17]). This indicates that a biochemical oscillator with a homeostatic period against an environmental change can easily shift its phase under the same environmental change. We also confirmed such reciprocity against change in the concentration of ATP (adenosine triphosphate), i.e., the case of nutrient compensation (see Fig. 5 of Ref. [17]).

Now, we examine if such reciprocity holds in the other class of circadian clocks, the TTO. In the TTO model, a clock-related gene is first transcribed and translated, and later such a translated protein represses the expression of its own gene with a time delay. When the transcription rate decreases, the amount of such a protein also decreases, which weakens the suppression of the clock-related gene expression. Consequently, such genes are transcribed again, leading to the oscillation of the gene expression level. As a typical example of the TTO model, we choose 


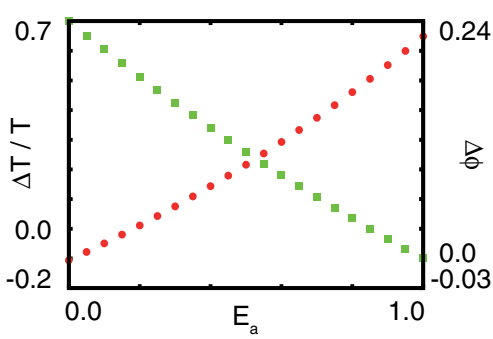

FIG. 2 (color online). Reciprocity between the robustness of period and plasticity of phase in the TTO model. Difference between periods at two temperatures $\left(\beta_{1}=0.0\right.$ and $\left.\beta_{2}=0.5\right)$ $(\Delta T / T$, red circle) and the amplitude of the phase response curve against a transient jump of temperature from $\beta_{1}$ to $\beta_{2}(\Delta \phi$, green square) plotted against various $E_{a}$, while activation energies for other reactions are fixed at 1.0. $\Delta T / T$ and $\Delta \phi$ are calculated similarly to how they are calculated in Fig. 1.

here a model of the circadian clock of a fruit fly [22] (see model equations and Fig. 1(b) of Ref. [17]). By varying the activation energy for mRNA degradation, $E_{a}$, and fixing activation energies for other reactions, we measured $\Delta \phi$ and $\Delta T / T$ using the same procedure as in the Kai model. Then, $\Delta T / T$ is low and $\Delta \phi$ is high for a low $E_{a}$ value, and $\Delta T / T(\Delta \phi)$ increases (decreases) with the increase in $E_{a}$ (Fig. 2 and see also Fig. 7 of Ref. [17]). Thus, the reciprocity holds also in the TTO.

To discuss the reciprocity analytically, we then study the Stuart-Landau model, a minimal model for simple sinusoidal oscillation [23]. The model consists of the amplitude $R$ and argument $\Theta$, where $R$ and $\dot{\Theta}$ reach a constant value at the limit-cycle attractor. Indeed, this model is derived as a normal form close to the Hopf bifurcation point. We introduce an external parameter $\beta$ :

$$
\begin{aligned}
& \frac{d R(\beta)}{d t}=f_{1}(\beta) R-R^{3}, \\
& \frac{d \Theta(\beta)}{d t}=f_{1}(\beta) \omega+f_{2}(\beta) R^{2},
\end{aligned}
$$

where, $f_{1}(\beta)$ is a response function of the first order term in complex Ginzburg-Landau equation, and $f_{2}(\beta)$ is that of the third order term (for choice of each functions, see Ref. [17]). Considering the stability of the limit cycle, the relaxation of $R$ after perturbation is assumed to be much faster than that of $\Theta$ [24]. Here, the period is given as

$$
T(\beta)=2 \pi\left\{f_{1}(\beta)\left[\omega+f_{2}(\beta)\right]\right\}^{-1} .
$$

Thus, after the change $\beta \rightarrow \beta+\Delta \beta$, the dependence of period on $\beta$ is given as

$$
\Delta \ln T(\beta) \simeq-\Delta \ln f_{1}(\beta)-\Delta f_{2}(\beta)\left[\omega+f_{2}(\beta)\right]^{-1} .
$$

Here, we neglected higher order terms of $\Delta \beta$, assuming that it is sufficiently smaller than $\beta$. From Eq. (3), if $\Delta \ln f_{1}(\beta)=-\Delta f_{2}(\beta)\left[\omega+f_{2}(\beta)\right]^{-1} \quad$ [i.e., $f_{1}^{\prime} / f_{1}=-f_{2}^{\prime} /$ $\left.\left(\omega+f_{2}\right)\right]$ is satisfied, the dependence of the period on $f_{2}(\beta)$ will be counterbalanced by $f_{1}(\beta)$, and the period is compensated against a change in $\beta$.

The argument $\Theta$ is defined only on a limit-cycle orbit, and we introduce the phase $\phi$ to extend the definition to the phase space out of the limit-cycle attractor, in particular to its basin. It is postulated that $\phi$ agrees with $\Theta$ on the limit-cycle orbit, i.e., different orbits from the same $\phi$ converge to the same point on the limit cycle having the same $\Theta$ value. Now, we will derive an isochrone, which is a set of points with the same $\phi$ on the phase space. $\phi$ is expected to have rotational symmetry; hence, the isochrone of the Stuart-Landau equation against the parameter $\beta$ is derived as

$$
\phi(R, \Theta, \beta)=\Theta+f_{2}(\beta)\left\{\ln R-\frac{1}{2} \ln f_{1}(\beta)\right\} .
$$

(see the Supplemental Material in Ref. [17].) Then, we consider an operation that increases $\beta$ from $\beta_{0}$ to $\beta_{0}+\Delta \beta$ and instantaneously reverses it to $\beta_{0}$. By assuming that $R$ instantaneously relaxes to $R^{*}\left(\beta_{0}+\Delta \beta\right)=\left[f_{1}\left(\beta_{0}+\Delta \beta\right)\right]^{1 / 2}$ while $\Theta$ remains unchanged, the phase after the above operation is derived as

$$
\begin{aligned}
\phi\left(\beta_{0}+\Delta \beta\right)= & \Theta\left(\beta_{0}\right) \\
& +\frac{f_{2}\left(\beta_{0}\right)}{2}\left\{\ln f_{1}\left(\beta_{0}+\Delta \beta\right)-\ln f_{1}\left(\beta_{0}\right)\right\} .
\end{aligned}
$$

Hence, when $\Delta \beta \ll \beta$, the change in phase is derived as

$$
\Delta \phi\left(\beta_{0}\right)=f_{2}\left(\beta_{0}\right) \Delta \ln f_{1}(\beta) / 2 .
$$

Therefore, from Eqs. (3) and (6), changes in the period and phase are represented by an equality.

$$
a \Delta \ln T+\Delta \phi=c,
$$

where $\quad a=f_{2}(\beta) / 2, \quad c=-f_{2}(\beta) \Delta f_{2}(\beta) / 2\left[\omega+f_{2}(\beta)\right]$, which depend only on $f_{2}(\beta)$ and not on $f_{1}(\beta)$. Thus, when we construct $f_{1}(\beta)$, which compensates for the dependence of $f_{2}(\beta)$ on $\beta$ according to Eq. (3), the phase is altered as $\Delta \phi=c$. On the other hand, when $f_{1}(\beta)$ is independent of $\beta$, the phase is also independent due to Eq. (6), while the period is strongly dependent on $\beta$ as $\Delta \ln T=c / a=-\Delta f_{2}(\beta) /$ $\left[\omega+f_{2}(\beta)\right]$.

We also confirmed the reciprocity is valid in the modified van der Pol oscillator [25] with strong nonlinearlity, i.e., beyond the neighborhood of Hopf bifurcation (See Fig. 9 of Ref. [17]).

The origin of reciprocity is also understood from the viewpoint of the adaptation motif. The standard minimal feedforward motif for adaptation consists of two components, $x$ and $y$ [26]. In the feedforward network in Fig. 3(a), an input changes both components $x$ and $y$, while $y$ gives an input to $x$. Here, the direct path to $x$ and the indirect path via 


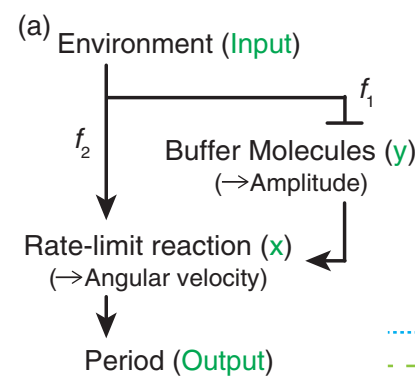

(b)

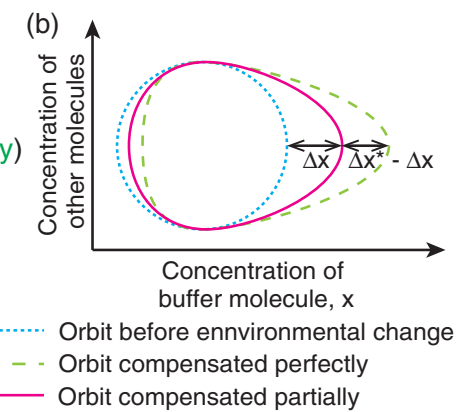

FIG. 3 (color online). Schemes of the reciprocity between the robustness of period and plasticity of phase. (a) Schematic networks of a generic (bio)chemical oscillator exhibiting homeostasis of period. Pointed and flat arrowheads indicate positive and negative regulation, respectively. Correspondences with a simple feedforward adaptation motif are represented by green characters in parentheses. (b) Scheme of limit-cycle orbits with compensation of the period against environmental change. Blue dotted line is a stable limit-cycle orbit before environmental change. The green dashed line and magenta solid line are stable limit-cycle orbits after environmental change when the period is perfectly compensated and partially compensated, respectively.

$y$ from the input have opposite signs. Then, the response of the output $x$ via the direct path is later canceled by $y$, and the adaptation behavior against the input is shaped. The degree of adaptation depends on the strength of the indirect regulation; weak regulation induces a partial adaptation and strong regulation leading to the cancellation of the two paths, induces perfect adaptation [27,28].

Our Stuart-Landau model also has a feedforward motif consisting of amplitude and angular velocity. When an environmental condition $\beta$ is changed, the angular velocity and amplitude are altered by the terms $f_{1}(\beta)$ and $f_{2}(\beta)$. After a direct change in angular velocity, the change is relaxed by the change in amplitude. The period is determined as the inverse of the angular velocity. If changes in the amplitude are large, the period is perfectly compensated and the phase is plastic. In contrast, if the change in amplitude is small, the angular velocity shows partial adaptation leading to partial compensation of the period while the phase is only slightly altered. Therefore, the reciprocity is understood as the adaptation dynamics on a limit cycle.

Indeed, the above argument of the adaptation on the limit cycle generally holds, for PTO and TTO models, where we can generally consider the scheme of Fig. 3(a). Environmental change directly influences the angular velocity while it is also buffered in the amplitude and then influences the phase. In a biochemical clock, the period mainly depends on the rate-limit reactions, which are slower than others. Environmental change will alter the speed of such rate-limit reactions, which is later counterbalanced by the change in the concentration of buffer molecules [29]. In fact, in the PTO model, the amount of free enzyme working as a buffer molecule can counterbalance the speed of the rate-limit reaction. Hence, the period of the oscillator is homeostatic against environmental changes. Likewise, in a TTO model, mRNA plays the role of such a buffer molecule.

Then, the limit-cycle orbit of oscillators with compensation shifts in the phase space of chemical concentrations to change that of a buffer molecule [see Fig. 3(b)]. When homeostatic response is achieved, the concentration of a buffer molecule $x$ should be changed with $\Delta x$ by the change in the external environment. Then the limit-cycle orbit will be shifted to change the concentration of a buffer molecule, and the magnitude of such a shift and the change in isocline will be $O(\Delta x)$ considering the continuous change in the isocline against $\Delta x$ which is small. Then, $\Delta \phi \propto \Delta x$ is expected. On the other hand, when the change in the concentration of a buffer molecule is not sufficient to counterbalance the environmental stimulus, the concentrations of other molecules will change. Let us represent the concentration of $x$ needed for perfect adaptation as $\Delta x^{*}$. Then, the change in the concentration of the other molecule of the lowest order is proportional to $\Delta x^{*}-\Delta x$. The period also changes accordingly, so that $\Delta T / T \propto \Delta x^{*}-\Delta x$ is expected. By combining the two proportional relationships, we obtain $a \Delta \phi+b \Delta T / T=\Delta x^{*}$ with coefficients of proportionality $a$ and $b$.

We have shown that reciprocity exists in both the PTO and TTO models. The currently known mechanisms of circadian oscillation can be classified into the above two cases [15], and the reciprocity is expected to be achieved universally in circadian clocks [31]. In a circadian clock system of a mold, Neurospora crassa, it was reported that a loss-of-temperature-compensation mutant, frq-7, shows smaller phase shift against transient temperature change than the wild type $[8,37,38]$. Although the quantitative relationship between temperature compensation and phase plasticity was not investigated therein, we expect that a quantitative experiment will confirm our reciprocity, not only in Neurospora crassa but also in other organisms in which loss-of-temperature-compensation mutants are isolated, e.g., fruit fly [39] and cyanobacteria [40]. Here, we demonstrated the reciprocity against changes in the temperature and the nutrient concentration, but from a theoretical consideration, it is expected to hold generally against a variety of stimuli, such as the change in strength of light and transcription rate [41,42], as long as the adaptation mechanism works. Moreover, it is also expected that the reciprocity is not limited to the circadian clock; it holds generally as long as the adaptation mechanism with buffering molecules works [43]. Our reciprocity will give a general quantitative law for such adaptation systems.

This work was partially supported by the Platform for Dynamic Approaches to Living System from MEXT, Japan; Dynamical Micro-scale Reaction Environment Project, JST; and JSPS KAKENHI Grant No. 15K18512. The authors would like to thank B. Pfeuty, H. Kori, K. Fujimoto, and U. Alon for useful discussion. 
*hatakeyama@complex.c.u-tokyo.ac.jp

[1] J. C. Dunlap, Cell 96, 271 (1999).

[2] D. Bell-Pedersen, V. M. Cassone, D. J. Earnest, S. S. Golden, P.E. Hardin, T. L. Thomas, and M. J. Zoran, Nat. Rev. Genet. 6, 544 (2005).

[3] C. S. Pittendrigh, Proc. Natl. Acad. Sci. U.S.A. 40, 1018 (1954).

[4] J. Hastings and B. Sweeney, Proc. Natl. Acad. Sci. U.S.A. 43, 804 (1957).

[5] C. S. Pittendrigh and S. Daan, J. Comp. Physiol. A 106, 223 (1976).

[6] C. S. Pittendrigh and S. Daan, Science 186, 548 (1974).

[7] W. F. Zimmerman, C.S. Pittendrigh, and T. Pavlidis, J. Insect Physiol. 14, 669 (1968).

[8] P. L. Lakin-Thomas, G. G. Coté, and S. Brody, Crit. Rev. Microbiol. 17, 365 (1990).

[9] M. Nakajima et al., Science 308, 414 (2005).

[10] T. Yoshida, Y. Murayama, H. Ito, H. Kageyama, and T. Kondo, Proc. Natl. Acad. Sci. U.S.A. 106, 1648 (2009).

[11] D. A. Rand, B. V. Shulgin, J. D. Salazar, and A. J. Millar, J. Theor. Biol. 238, 616 (2006).

[12] T. Takeuchi, T. Hinohara, G. Kurosawa, and K. Uchida, J. Theor. Biol. 246, 195 (2007).

[13] O. E. Akman, D. A. Rand, P. E. Brown, and A. J. Millar, BMC Syst. Biol. 4, 88 (2010).

[14] J. Tomita, M. Nakajima, T. Kondo, and H. Iwasaki, Science 307, 251 (2005).

[15] X. Qin, M. Byrne, Y. Xu, T. Mori, and C. H. Johnson, PLoS Biol. 8, e1000394 (2010).

[16] J. S. van Zon, D. K. Lubensky, P. R. H. Altena, and P. R. ten Wolde, Proc. Natl. Acad. Sci. U.S.A. 104, 7420 (2007).

[17] See Supplemental Material at http://link.aps.org/ supplemental/10.1103/PhysRevLett.115.218101 for detailed information of models, and analysis of Stuart-Landau model and van der Pol oscillator.

[18] T. S. Hatakeyama and K. Kaneko, Proc. Natl. Acad. Sci. U.S.A. 109, 8109 (2012).

[19] T. S. Hatakeyama and K. Kaneko, FEBS Lett. 588, 2282 (2014).

[20] A. T. Winfree, The Geometry of Biological Time (Springer, Berlin, 1980).

[21] $\Delta \phi$ is thought to be less model dependent than the other measures including the entrainability and the shape of the PRC.

[22] G. Kurosawa and Y. Iwasa, J. Theor. Biol. 233, 453 (2005).

[23] Y. Kuramoto, Chemical Oscillations, Waves, and Turbulence (Springer, Berlin, 1984).
[24] If the system is in the vicinity of Hopf bifurcation, the relaxation of $R$ may be slowed down, and further study is necessary.

[25] B. van der Pol, London, Edinburgh Dublin Philos. Mag. J. Sci. 2, 978 (1926).

[26] U. Alon, An Introduction to Systems Biology: Design Principles of Biological Circuits (CRC press, Florida, 2006).

[27] D. E. Koshland, A. Goldbeter, and J. B. Stock, Science 217, 220 (1982).

[28] L. A. Segel, A. Goldbeter, P. N. Devreotes, and B. E. Knox, J. Theor. Biol. 120, 151 (1986).

[29] Specific molecules for the buffering mechanism for the robustness of the period of biological clocks depend on a particular target system. Still, as long as the mechanism exists, the reciprocity holds. Indeed, in the in silico evolution of a circadian clock, such a buffer molecule naturally evolves [30].

[30] P. François, N. Despierre, and E. D. Siggia, PLoS Comput. Biol. 8, e1002585 (2012).

[31] Our result implies the negative correlation between the robustness of the period and the variation of entrainment phase [5,32-35], as the latter was reported to be a negative correlation with the amplitude of the PRC [36].

[32] J. Aschoff and H. Pohl, Naturwissenschaften 65, 80 (1978).

[33] T Roenneberg, A Wirz-Justice, and M Merrow, J. Biol. Rhythms 18, 80 (2003).

[34] C. Gronfier, K. P. Wright, R. E. Kronauer, and C. A. Czeisler, Proc. Natl. Acad. Sci. U.S.A. 104, 9081 (2007).

[35] U. Abraham, A. E. Granada, P. O. Westermark, M. Heine, A. Kramer, and H. Herzel, Mol. Syst. Biol. 6, 438 (2010).

[36] A. E. Granada, G. Bordyugov, A. Kramer, and H. Herzel, PLoS One 8, e59464 (2013).

[37] H. Nakashima, Journal of interdisciplinary cycle research 18, 1 (1987).

[38] L. Rensing, A. Bos, J. Kroeger, and G. Cornelius, Chronobiology international 4, 543 (1987).

[39] A. Matsumoto, K. Tomioka, Y. Chiba, and T. Tanimura, Mol. Cell. Biol. 19, 4343 (1999).

[40] Y. Murayama et al., EMBO J. 30, 68 (2011).

[41] C. Dibner, D. Sage, M. Unser, C. Bauer, T. dEysmond, F. Naef, and U. Schibler, EMBO J. 28, 123 (2009).

[42] J. K. Kim and D. B. Forger, Mol. Syst. Biol. 8, 630 (2012).

[43] In weakly coupled oscillators under small perturbation or in the vicinity of Hopf bifurcation, robustness of the period can be achieved without the adaptation mechanism. Such oscillators without the adaptation mechanisms do not (necessarily) satisfy the reciprocity. 